\title{
The autoclaving and re-implantation of an infected prosthesis as a spacer during resection knee arthroplasty: a systematic review
}

\author{
Antonio Spinarelli ${ }^{1} \cdot$ Davide Bizzoca $^{1,3}$ (D) $\cdot$ Lorenzo Moretti $^{1} \cdot$ Giovanni Vicenti $^{1} \cdot$ Raffaele Garofalo $^{2} \cdot$ Biagio Moretti $^{1}$
}

Received: 31 January 2021 / Accepted: 12 July 2021 / Published online: 28 July 2021

(c) The Author(s) 2021

\begin{abstract}
Background Hofmann et al., in 1995, first described an articulating spacer made by cleaning and autoclaving the original femoral component, which is then re-implanted with a new tibial polyethylene. This systematic review aims to assess the state of existing evidence on the intraoperative autoclaving and re-use of an infected prosthesis, as a spacer, during a twostage revision following Periprosthetic Joint Infections (PJI).

Methods A systematic review was conducted with methods described in the Preferred Reporting Items for Systematic Reviews and Meta-Analyses. OVID-MEDLINE ${ }^{\circledR}$, EMBASE, Cochrane Library, SCOPUS, Web of Science, Google Scholar and PubMed were searched from 1995 to April 2020 to identify relevant studies.

Results Fourteen studies were included in this systematic review: two prospective case series; six retrospective comparative studies and six retrospective case series. The reviewed studies included 567 patients (571 knees): 394 patients treated with autoclaved components and 173 with a spacer made of new components. The cumulative re-infection rate in patients treated with re-used autoclaved components was 13.7\% (54 re-infections in 394 patients), whereas in control patients the re-infection rate was $13.3 \%$ ( 23 re-infections in 173 patients). The final Range of Movement in patients treated using the autoclaved components as a spacer, compared with patients receiving static spacers, was significantly higher in three out of four comparative studies.

Conclusion There is a moderate level of evidence that the intraoperative autoclaving and re-use of an infected prosthesis as a spacer, during a knee resection arthroplasty, is an effective procedure in the management of knee PJI.
\end{abstract}

Keywords Periprosthetic joint infection · Total knee replacement · Two-stage revision strategy $\cdot$ Autoclaving $\cdot$ Re-use $\cdot$ Knee osteoarthritis

Davide Bizzoca

da.bizzoca@gmail.com

1 Neuroscience and Sense Organs, Orthopaedic and Trauma UnitDepartment of Basic Medical SciencesSchool of Medicine, University of Bari Aldo Moro, AOU Consorziale "Policlinico", Piazza Giulio Cesare 11, 700124 Bari, Italy

2 Upper Limb Unit. "F Miulli” Hospital, Acquaviva Delle Fonti, Bari, Italy

3 PhD Course in Public Health, Clinical Medicine, and Oncology, University of Bari "Aldo Moro, Piazza Giulio Cesare 11, 70100 Bari, Italy

\section{Introduction}

Periprosthetic Joint Infection (PJI) is currently one of the most dreadful complications following total joint replacement (TJR) [1].

It is reported that PJI is the third most common cause of Total Hip Arthroplasty (THA) failure and the leading reason for Total Knee Arthroplasty (TKA) failure, accounting for $14.8 \%$ and $16.8 \%$ of all hip and knee revisions, respectively [1-3]. Nonetheless, a substantial increase in the prevalence of PJI is expected in the next years, mainly due to the increasing volume of TJRs performed all over the world, the emergence of resistant microorganisms and the tendency to perform joint arthroplasty even in patients with extensive comorbidities [4-6, 44, 45].

PJIs have a significant impact on the patient's health status and quality of life, since they may cause severe pain, 
a progressive restriction of movement, feelings of isolation, depression, hopelessness and, if incorrectly managed, also a lethal epilogue [7, 8]. Thus, the Orthopedic community is paying great attention to the study and the treatment of this disease.

PJI may be classified, according to Zimmerli et al., into early, delayed and chronic infections [9]. Early PJIs occur within 3 months after TJR, whereas PJIs with onset between 3 and 24 months are classified as delayed infections and those occurring more than 24 months after TJR are classified as late [9].

In the management of chronic PJI, the two-stage revision strategy has evolved as the gold standard and preferred procedure, with a success rate exceeding $90 \%$ $[10,11]$. In this procedure, the first step is the removal of infected prosthetic components and the concomitant implant of a cement spacer, followed by a period of tailored systemic antibiotic therapy $[10,11]$. The re-implantation of revision components is then performed when the normalization of blood tests, synovial fluid analysis and local clinical signs of PJI are detected [10, 11]. Three meta-analyses have recently investigated the effectiveness of the two-stage surgical revision of the infected THA [8, 12] and TKA [13], compared with one-stage revision strategy, and both procedures resulted effective in the treatment of PJI in generally unselected patients [8, 12, 13].

Cement spacers are used in the first step of revision arthroplasty to maintain the joint space in distraction while providing high-dose local antibiotic delivery [11, 14]. They could be classified as static and articulating antibiotic-loaded spacers; it is reported that there is no significant difference between a non-articulating and an articulating spacer, in the treatment of PJI.

Static spacers, however, prevent joint movements until the second stage, and thus increase patient discomfort and may cause soft tissue contracture [15]. Therefore, articulating cement spacers have been developed to allow the patient to perform joint movements to some extent before the second-stage revision arthroplasty, therefore also preventing soft tissue contracture $[16,17]$.

Different types of articulating spacers have been described, including metal-on-polyethylene, cement-oncement, or cement-on-polyethylene spacers [16, 17].

Hofmann et al. [18], in 1995, first described the treatment of an infected TKA using an articulating spacer made by cleaning and autoclaving the original femoral component. The autoclaved component was then re-implanted, with a new tibial polyethylene liner. These components are cemented into place using antibiotic-impregnated bone cement. To date, several studies have investigated the effectiveness of such a kind of spacer, but no randomized controlled trials have been conducted on these subjects.
This systematic review aims to assess the state of existing evidence on the intraoperative autoclaving and re-use of an infected prosthesis, as a spacer, during a knee resection arthroplasty performed for PJI.

\section{Methods}

The study was conducted with methods described in the Preferred Reporting Items for Systematic Reviews and MetaAnalyses (PRISMA) [20, 21].

\section{Search strategy}

OVID-MEDLINE®, EMBASE, Cochrane Library, SCOPUS, Web of Science, Google Scholar and PubMed were searched from 1995 to April 2020 to identify relevant studies for further analysis.

The main keywords were: "autoclaved" or "autoclaving" and "component" and "re-use", or "periprosthetic joint infection", or "total knee replacement", or "total knee arthroplasty", or "two-stage re-implantation", or "articulating spacer". A manual search of the reference lists of the selected publications was also performed, to identify additional studies for potential inclusion.

One review Author (BM) scanned the titles and abstracts. Potentially relevant articles were acquired for full-length text and Authors were contacted when the article was not available.

\section{Eligibility criteria}

Full-text articles alone published between December 1995 and April 2020 were included. The review was restricted to articles published in English.

Inclusion criteria were: (1) all study designs; (2) detailed autoclaving procedure of the infected components; (3) sufficient data presented to estimate the re-infection rate and to assess the final clinical outcome.

Exclusion criteria were: (1) less than one year of followup, (2) re-use of components treated with other procedures than autoclaving and (3) lack of data about microorganism identification.

\section{Data extraction}

Information was extracted from each study by one review Author (DB) and checked by another Author (AS), including: (1) characteristics of study participants (age, gender, duration of symptoms, microorganisms, follow-up) and the study inclusion and exclusion criteria; (2) autoclaving protocol of the infected components; (3) surgical therapy and antimicrobial treatment regimen; (4) treatment failure 
definition; (5) number of patients meeting the inclusion criteria; (6) outcomes and (7) re-infection rate. Disagreements were resolved by discussion between them.

\section{Study quality and risk of bias of the studies}

The quality of each included study was assessed according to the AAOS clinical practice guideline and review methodology version 2, (available at www.orthoguides.org). The following points were evaluated: sample size and features; description of inclusion end exclusion criteria; blinding of participants and personnel (in randomized studies); appropriate statistical analysis; references of the study; data evaluation; the presence of bias; the presence of confounding factors; follow-up length.

Based on the depicted flaws and the study design, the quality of each study was defined as follows:

1. Prognostic study: high-quality study ( $<1$ flaw); moderate-quality study ( $\geq 1$ and $<2$ flaws); low-quality study ( $\geq 2$ and $<3$ flaws) and very low-quality study ( $\geq 3$ flaws).

2. Diagnostic study: high-quality study ( $<1$ flaw); moderate-quality study ( $\geq 1$ and $<2$ flaws); low-quality study ( $\geq 2$ and $<3$ flaws) and very low-quality study ( $\geq 3$ flaws).

3. Randomized study: high-quality study ( $<2$ flaws); moderate-quality study ( $\geq 2$ and $<4$ flaws); low-quality study ( $\geq 4$ and $<6$ flaws) and very low-quality study ( $\geq 6$ flaws).

4. Observational study: high-quality study ( $<2$ flaws); moderate-quality study ( $\geq 2$ and $<4$ flaws); low-quality study ( $\geq 4$ and $<6$ flaws) and very low-quality study ( $\geq 6$ flaws).

Two authors (L.M. and V. G.) independently evaluated all the studies. In case of disagreement between them, a new combined evaluation was performed.

The surgical procedures, the antibiotic regimen and the outcome definitions were evaluated in the included studies. Publication bias could not be assessed by a funnel plot considering the very low number of patients in each study.

\section{Primary outcome, secondary outcome}

The primary outcome was to assess the re-infection rate in patients undergoing a two-stage revision strategy for PJI, using an articular spacer made by autoclaving the infected components. The second aim was to assess the final and intermediate functional outcomes, in patients undergoing this procedure.

\section{Summary measures}

The cumulative re-infection rate was computed using extracted data from the relevant studies. It was defined as the number of re-infection during follow-up over the number of patients with chronic knee PJI treated with the two-stage revision strategy, using the autoclaved infected components as a spacer.

\section{Results}

\section{Study selection}

The OVID-MEDLINE®, EMBASE, Cochrane Library, SCOPUS, Web of Science, Google Scholar and PubMed database searches provided a total of 1,387 studies for potential inclusion in the review (Fig. 1). After adjusting for duplicates, 1,009 studies remained. Of these, 975 studies were discarded after reading titles and reviewing abstracts. The Cochrane Library provided no relevant studies. Three additional abstracts were identified by checking the references of the relevant papers.

The full text of the remaining 34 studies was examined in greater detail. Of these, 23 studies did not meet the inclusion criteria. Moreover, three additional studies, identified through a bibliographic cross-reference of the obtained articles, met the inclusion criteria. Therefore, fourteen studies were finally included in this systematic review [14-16, 22-29, 33-35].

\section{Study quality}

The process of quality assessment, performed according to the AAOS clinical practice guideline and review methodology version 2, depicted the following results: Four studies [15, 23-26] out of $14(28.57 \%)$ were classified as moderatequality studies, whereas 10 studies [14, 16, 22, 24, 27-29, 33-35] out of 14 (71.43\%) were classified as high-quality studies (Table 2).

Due to the low number of patients included in each study, publication bias could not be assessed.

\section{Study characteristics}

The study characteristics are summarized in Table- 1 and Table-2. Twelve retrospective studies and two prospective case series were included. Five hundred and sixty-seven patients (571 knees) were included in this review. The number of patients, gender, age, mean follow-up, microorganisms 


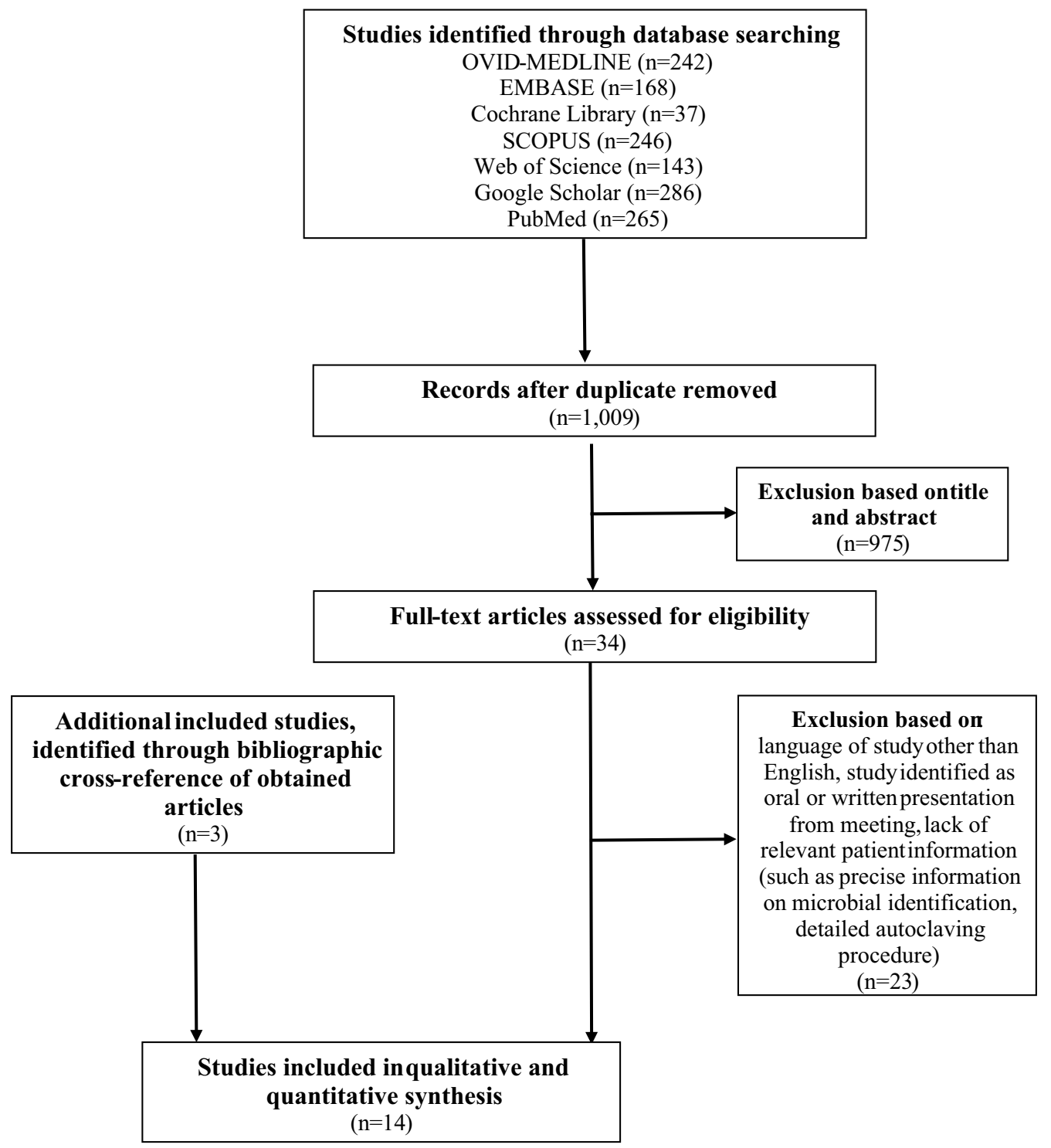

Fig. 1 PRISMA flow diagram

identification, the time between first and second-step revision, outcomes at the follow-up and re-infection rates is reported in Table 1.

Table 2 shows the autoclaving protocol, articular spacer details (the type of femoral component, type of tibial insert and antibiotic-impregnated cement features) and the antibiotic treatment performed. In all the studies, the patients underwent a two-stage revision strategy for knee PJI, using a spacer made by autoclaving the infected components. In six studies out of fourteen $(42.86 \%)$, the antibiotic-impregnated articular spacer was realized using an autoclaved femoral component and a new tibial polyethylene insert [15-22, 26-28, 34, 35]; in four studies out of fourteen $(28.57 \%$ ), both the femoral component and the tibial insert were autoclaved and re-used [14, 23-25] and in four studies out of fourteen $(28.57 \%$ ), a metal-oncement spacer was implanted [16, 27, 29, 33].

All the patients received an antibiotic-impregnated cement in the spacer, as well as adequate antibiotic therapy for at least five to six weeks (Table-2) [14-16, 22-29]. The autoclaving protocol was specified only in four studies out of fourteen $(28.57 \%)$ [16, 28, 29, 35]. Two studies out of fourteen $(14.28 \%)$ indicated only the duration time of the autoclaving process $[14,35]$, whereas it was not detailed in the remaining studies [22-27, 33, 34]. In all the studies, the implant was mechanically cleaned of all cement and tissue before undergoing autoclaving. 


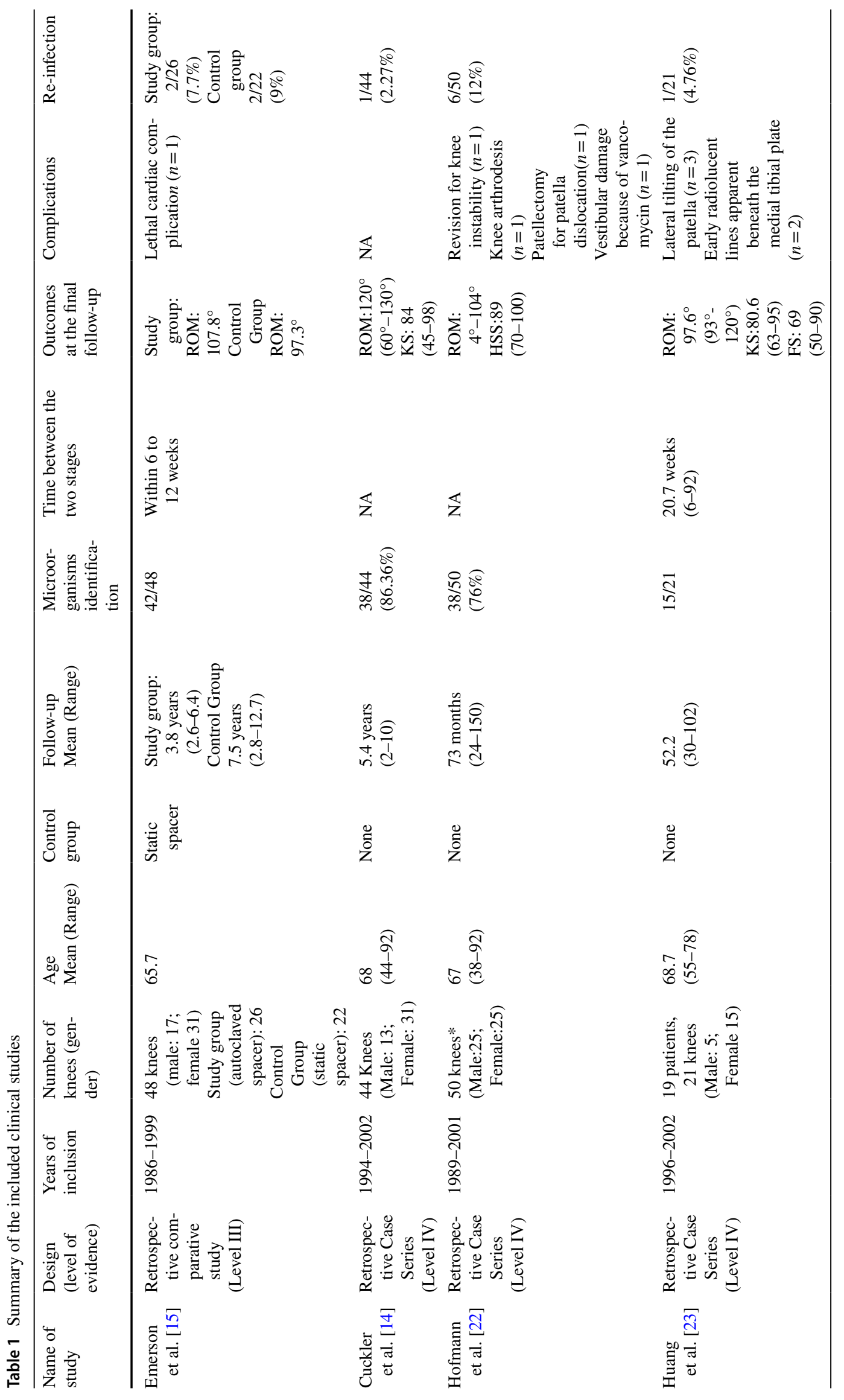




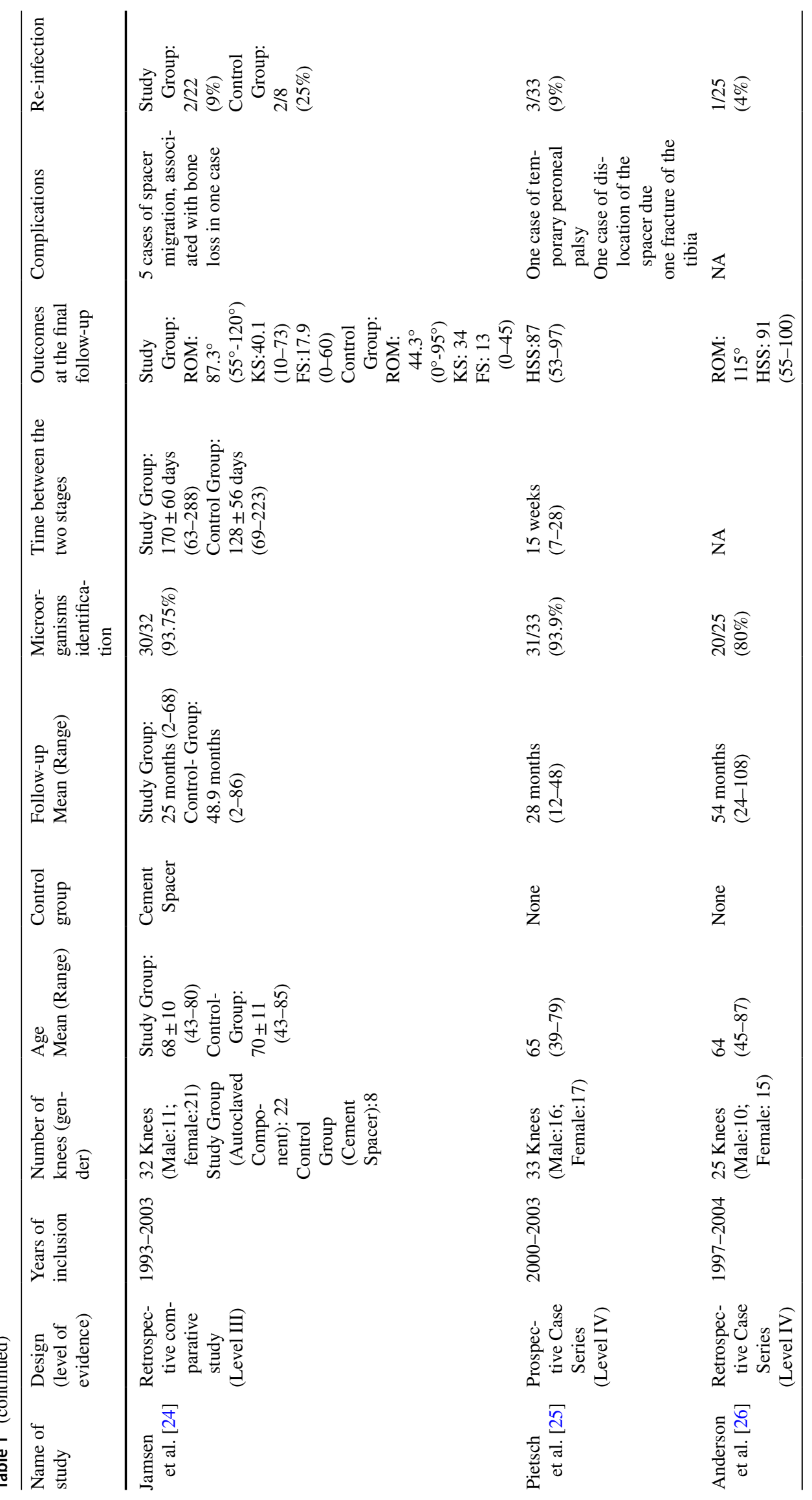




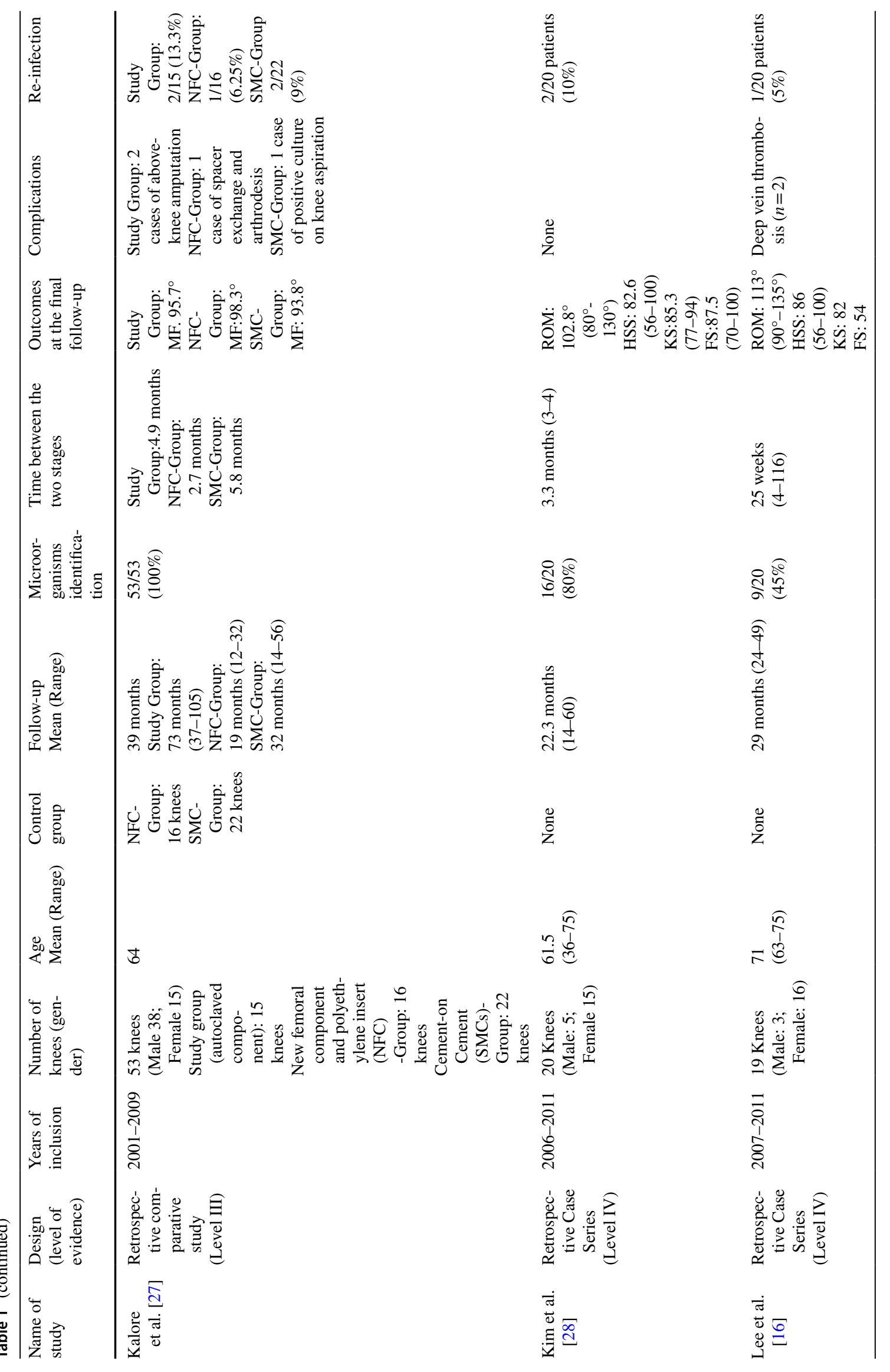




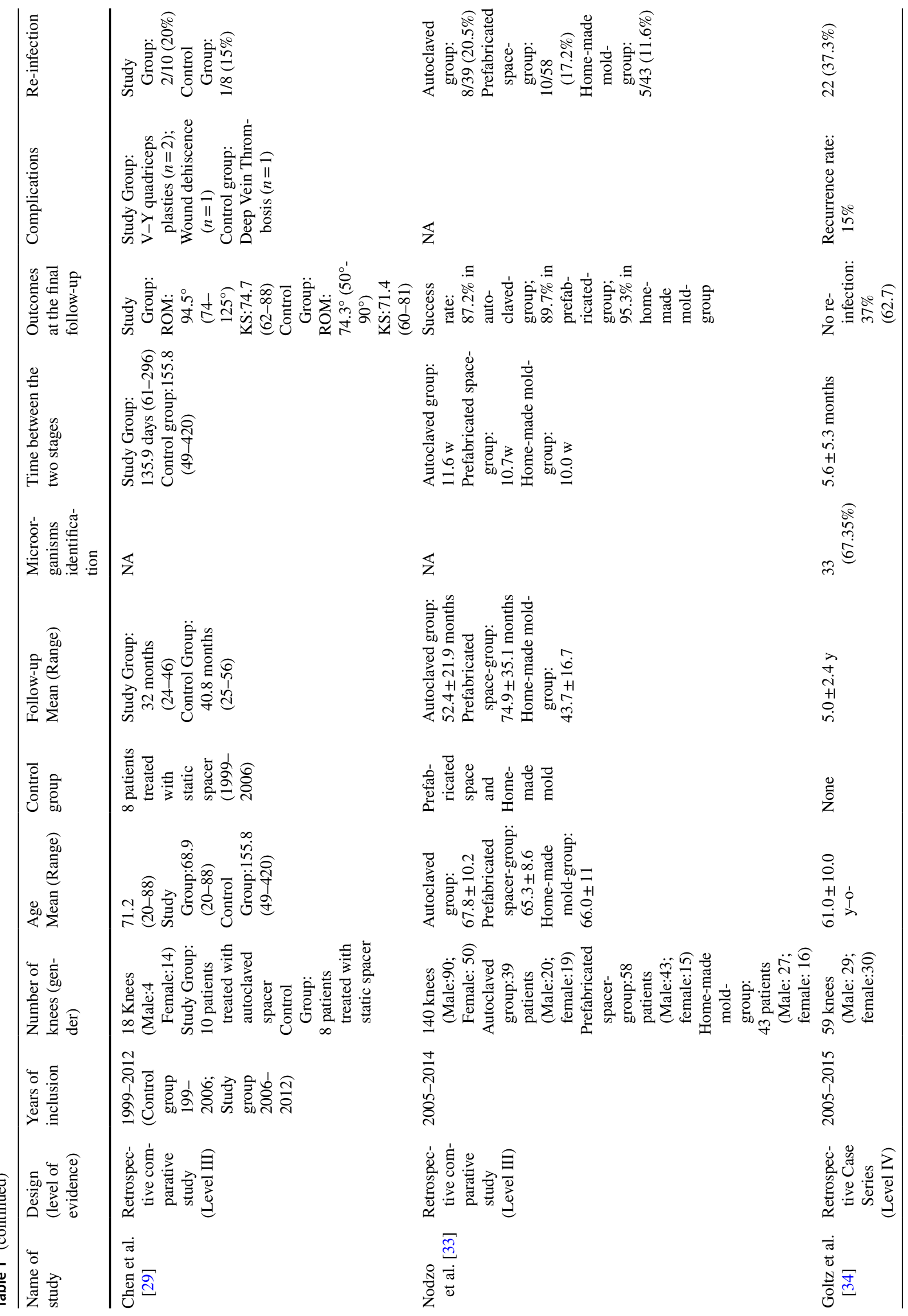




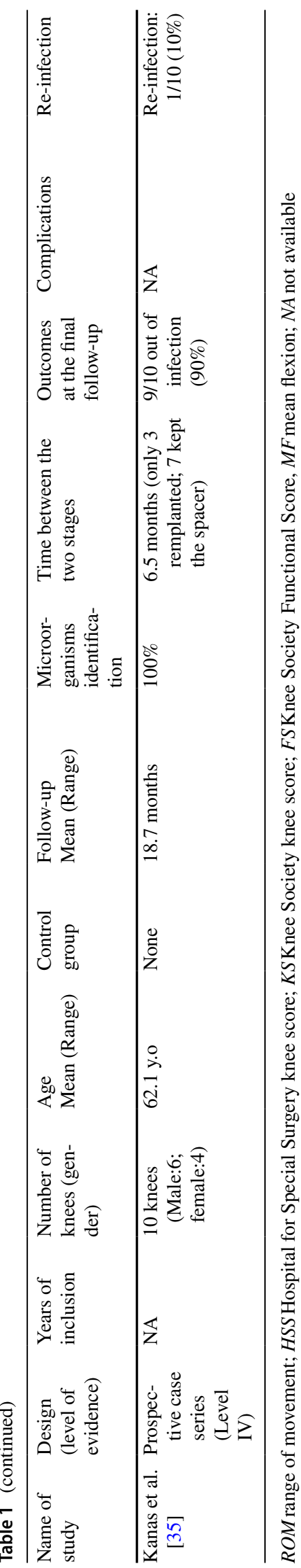

\section{The cumulative rate of re-infection}

The reviewed studies included 567 patients (571 knees): Three hundred and ninty-four patients treated with autoclaved components and 173 with a spacer made by new components. The cumulative re-infection rate in patients treated with re-used autoclaved components was 13.7\% (54 re-infections in 394 patients), whereas in control patients, the re-infection rate was $13.3 \%$ (23 re-infections in 173 patients).

\section{Mobile versus static articular spacers}

Patients treated with mobile articular spacers, made by autoclaving the infected components, showed, after the spacer implantation, a significant higher ROM, compared with those treated with static spacers, in one out of four comparative studies reviewed [27].

The final ROM in patients treated using the autoclaved components as a spacer, compared with subjects receiving static spacers, was significantly higher in three out of four comparative studies $[15,24,29]$. However, at final followup, the functional scores -i.e., Hospital for Special Surgery Knee Score (HSS), Knee Society Knee Score (KS) and Knee Society Functional Score (FS)-registered in patients treated with articular spacers, compared with static spacers group, showed no significant difference in all the reviewed comparative studies.

\section{Discussion}

\section{Summary of evidence}

PJI currently represents the leading cause of TKA failure and a further increase of prevalence is expected in future years [40-45]. Consequently, the management of an infected prosthesis is a hot topic in orthopedics.

Hofmann et al. [18], in 1995, first described the treatment of an infected TKA using an articulating spacer made by cleaning and autoclaving the original femoral component. This systematic review aims to assess the state of existing evidence on the intraoperative autoclaving and re-use of an infected prosthesis, as a spacer, during a knee resection arthroplasty performed for PJI.

The review results suggest that the intraoperative autoclaving and re-use of an infected prosthesis as a spacer, during a knee resection arthroplasty performed for PJI, is an effective strategy. Hence, a comparable re-infection rate was observed in patients managed with autoclaved components compared with patients treated with a new spacer. Moreover, patients receiving mobile articular spacers showed a better functional outcome at the final follow-up. 


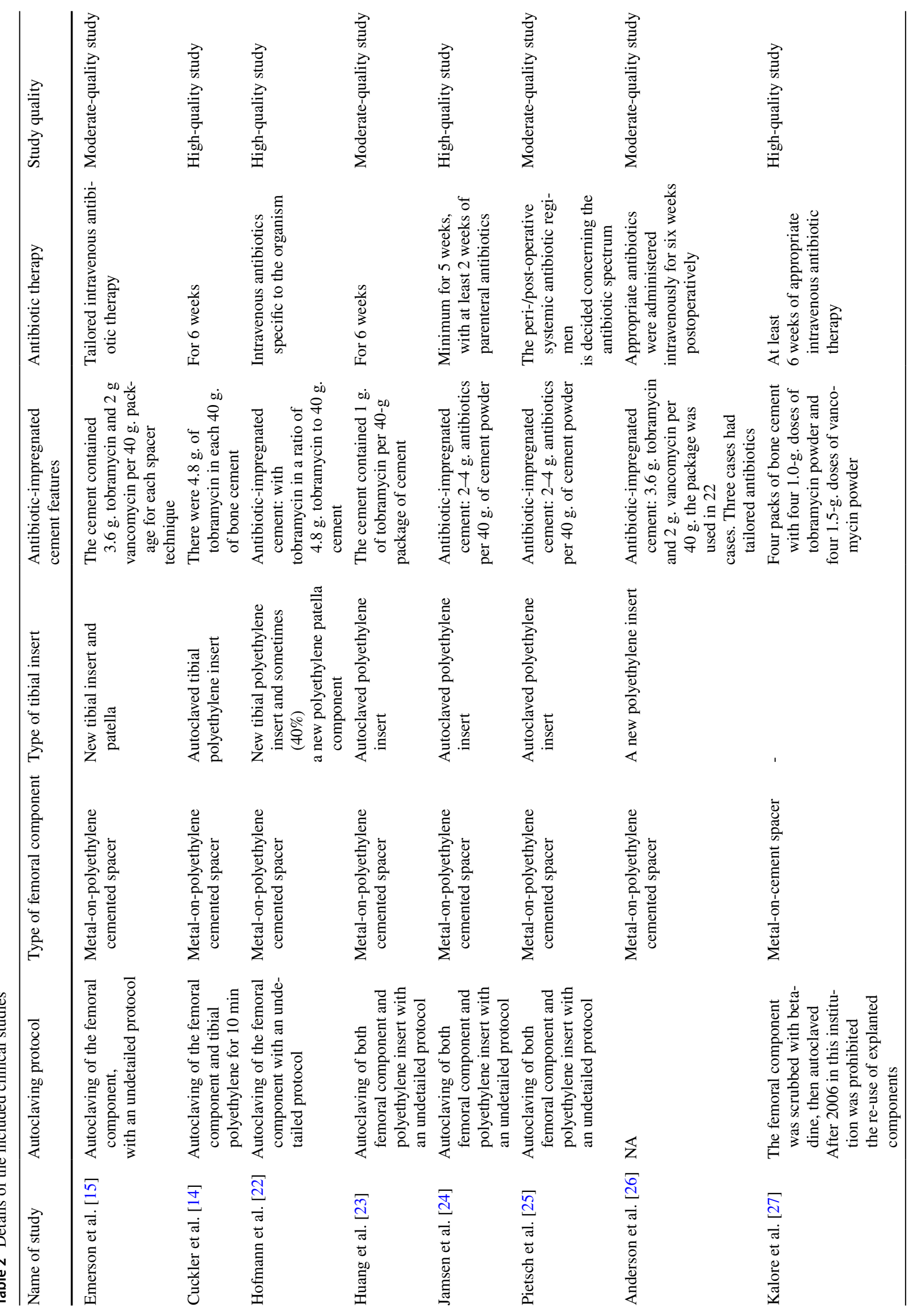




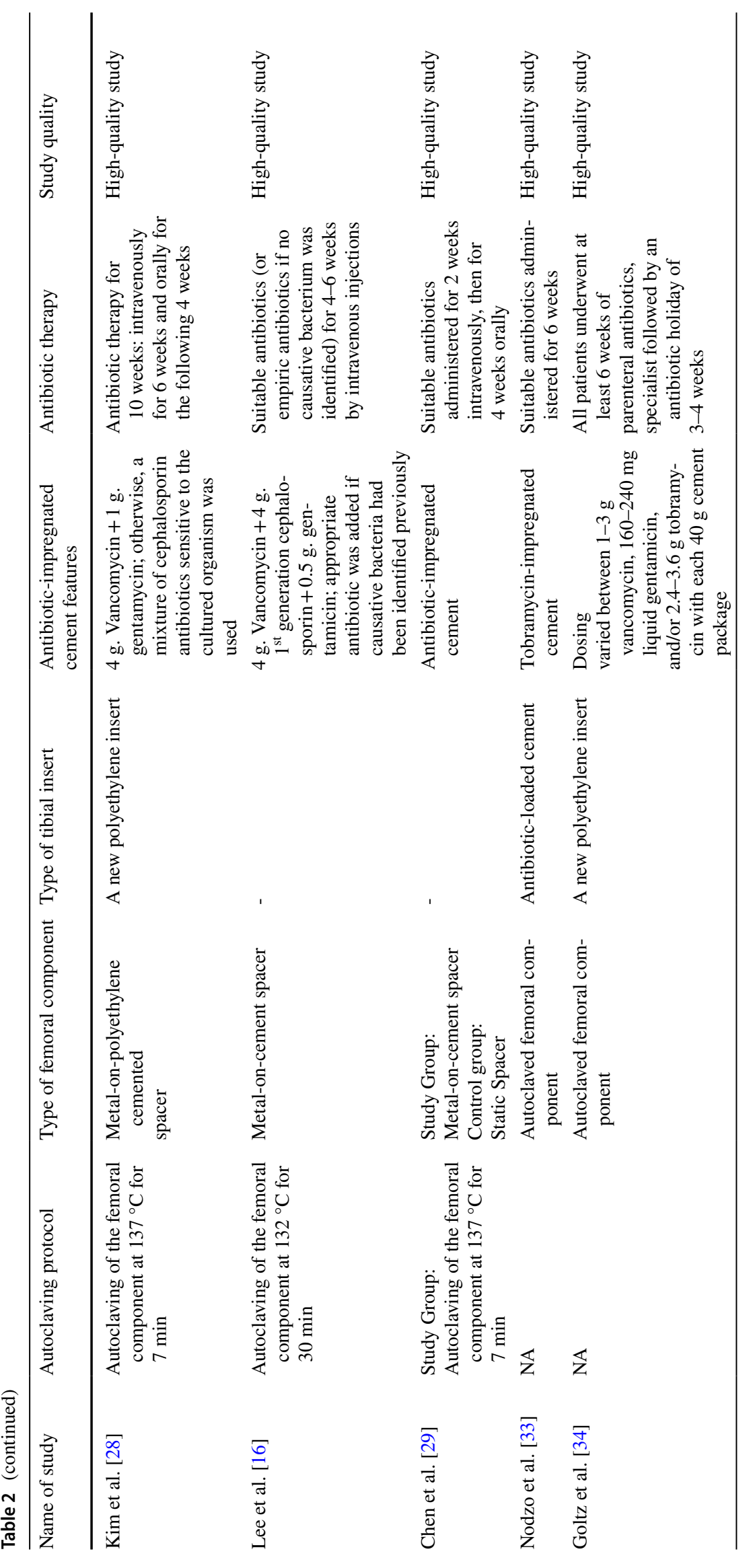




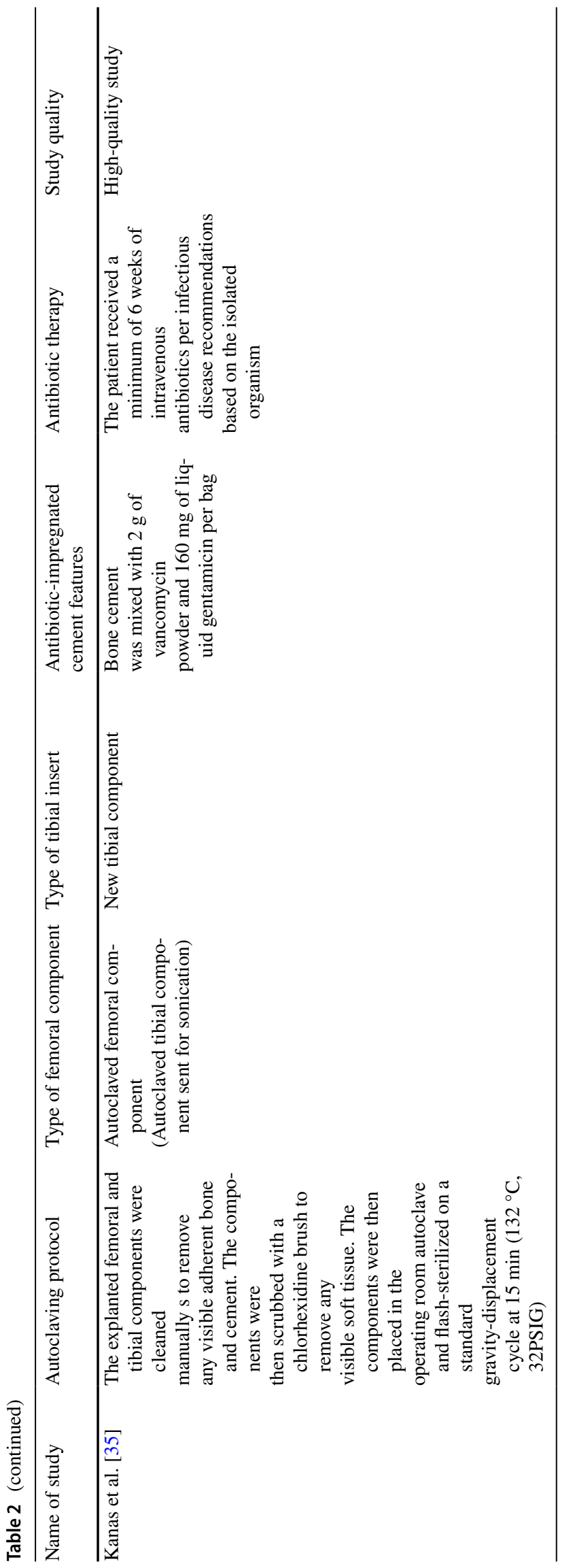

In this procedure, before undergoing autoclaving, the infected femoral component should be mechanically cleaned of all cement and tissue [30,37-39]. The autoclave should be near the operating room to facilitate aseptic delivery to the sterile field; the use of a rigid, re-usable sterilization container system is recommended [30]. If a spore test (it takes approximately one hour after the cycle) is not able to be run before implant use, then the implant should receive the equivalent of full-cycle steam sterilization and not a flash sterilization cycle [30].

Patients treated using this procedure showed a comparable re-infection rate to those undergoing a two-stage revision strategy, using a sterile cement spacer. The cumulative re-infection rate, at a minimum of two years follow-up, was $13.7 \%$ in patients treated with an autoclaved prosthesis and $13.3 \%$ in patients receiving a sterile cement spacer.

Pietsch et al. [25], in a prospective non-randomized trial on 33 patients with knee PJI undergoing two-stage revision using autoclaved both femoral component and polyethylene tibial insert, reported an infection rate of 9\% (3 re-infections out of 33) at a mean 28-month follow-up.

Kanas et al. [35] have recently performed a prospective case series, recruiting 10 patients with knee PJI. All the patients were managed with TKA explantation, debridement, and placement of an articulating antibiotic spacer consisting of the explanted and sterilized femoral component and a new polyethylene tibial insert [35]. Only 3 patients out of 10 were re-implanted, while the reaming 7 patients kept the spacer. At the final follow-up, a re-infection rate of $10 \%$ was observed [35].

In the retrospective comparative studies, the re-reinfection rates resulted not significantly different in patients treated with an autoclaved component with respect to those receiving new sterile spacers.

Emerson et al. [15], in a retrospective study comparing 26 patients treated with autoclaved components to 22 with sterile static spacers, reported a re-infection rate of $7.7 \%$ in the autoclaved components group (2 patients out of 26), at 3.8 years mean follow-up, and a re-infection rate of $9 \%$ (2 patients out of 22), at 7.5 years mean follow-up, in patients receiving sterile spacers. The Authors specified that there was not the same organism, in the re-infected patients treated with mobile spacers made by autoclaving the infected components [15].

Jamsen et al. [24] observed a re-infection rate of $9 \%$ (2 patients out of 22), at 25 months mean follow-up, in patients treated with re-sterilized prosthesis components and a reinfection rate of $25 \%$ ( 2 patients out of 8 ), at 48.9 months mean follow-up, in patients treated with cement spacers.

Kalore et al. [27] showed a $13.3 \%$ re-infection rate (2 patients out of 15), at mean 73-month follow-up, in patients operated on with autoclaved femoral component, a re-infection rate of $6.25 \%$ (1 patient out of 16), at a mean 19-month 
follow-up, in the group treated with a new femoral component and a $9 \%$ re-infection rate ( 2 patients out of 22 ), at mean 32-month follow-up, in subjects treated with silicone mold component spacers.

Chen et al. [29] reported a re-infection rate of $20 \%$ (2 patients out of 10), at 32-month mean follow-up, in patients treated with autoclaved femoral component and tibial insert and a re-infection rate of $15 \%$ (one patient out of 8 ), at 40.8 months mean follow-up, in those with sterile static spacers.

Nodzo et al. 2017 [33], in a retrospective comparative study including 140 patients with knee PJI, divided into three groups (i.e., autoclaved-group: 39 patients; prefabricated spacer-group:58 patients and home-made mold spacergroup: 43 patients), observed no statistically significant difference in the success rates between groups. A re-infection rate of $20.5 \%$ was observed in the autoclaved-group at the final follow-up.

This procedure has a good cost-effectiveness ratio, since it is reported that a spacer made by autoclaving the infected components has a direct cost of $\$ 932$, whereas spacers made by new femoral component cost $\$ 3589$ and molded cement spacers cost $\$ 3945$ [27]. It is also reported that the temporarily re-use of the femoral component can reduce the cost of the articulating spacer by approximately $\$ 1900 /$ case, versus a new femoral component, and by approximately $\$ 1000 /$ case, versus a molded cement spacer [30]. Consequently, this technique is a safe and cost-effective option to improve patient function during revision for PJI.

The value of these recommendations has been also confirmed by the data deriving from an in vitro and in vivo study [30]. Lyons et al. recently showed that six cobaltchrome femurs components, explanted from patients with knee PJI, became sterile after autoclaving under a standard gravity-displacement cycle $\left(132^{\circ} \mathrm{C}, 27 \mathrm{PSIG}, 10 \mathrm{~min}\right)$ [30].

Moreover, these Authors conducted an in vitro test on six sterile chrome cobalt femur implants ( 2 cementless, 4 cemented), inoculated with different bacterial species. After that, three of these components were autoclaved on a standard gravity-displacement vacuum cycle $\left(121^{\circ} \mathrm{C}, 15 \mathrm{PSIG}\right.$, $45 \mathrm{~min}$ ), while the remaining implants were maintained in a sterile environment at room temperature. All these components were subjected to $5 \mathrm{~min}$ of sonication; the diluted sonicate of the autoclaved components showed no bacterial growth on an agar plate, whereas the control components, that did not undergo autoclaving treatment after inoculation, highlighted growth of multiple colonies of the original bacteria [30]. Finally, the biofilm in vitro test of three MRSA biofilm covered cobalt-chrome pieces that underwent autoclave treatment, showed a statistically significant reduction of relative biofilm compared to controls [30]. The biofilm burden reduction was also confirmed by Scanning Electron Microscope images [30].
Interestingly, Park et al. [36] have evaluated the role of sonication in depicting the sterility of an autoclaved femoral component explanted from an infected TKA. These authors found only two infected femoral components out of nineteen $(10.53 \%)$ after sonification [36]. Hence, they concluded autoclaving of an infected femoral implant could be a good method for using the temporary articulating antibiotic spacer in two-stage revision arthroplasty [36].

Similar positive findings have been reported by Nodzo et al., in a prospective observational study [33]. These authors cleaned and autoclaved both the explanted femoral and tibial components. Then, the autoclaved femoral components were re-implanted, while the tibial ones were aseptically packaged and sent to a microbiology lab for sonication and culture of the sonicate for 14 days; all the cleaned tibial components were negative for bacterial growth of the infecting organism after final testing and analysis [33].

Unfortunately, the relatively low number of patients in our review, as well as the absence of randomized controlled trials among the reviewed articles, allow us to recommend a moderate level of evidence. Furthermore, it should be remarked that the Center for Disease Control (CDC), Association of Operating Room Nurses (AORN), health care institutions, implant companies and medical consult teams are hesitant to temporarily re-use implants for medical, legal and financial reasons [31, 32].

Moreover, other relevant concerns should be considered when performing this procedure, including a lack of guarantee of the re-used component sonication, the potential delayed surgical time because of the explanted component autoclaving and the off-label implant use, that might raise potential medicolegal issues.

It should be noted, however, that all the reviewed studies showed the re-use of the autoclaved component as a spacer is an effective procedure in the eradication of knee PJI.

\section{Strengths and limitations}

To our knowledge, this is the first systematic review, which aims to assess the role of intraoperative autoclaving and re-use of an infected prosthesis as a spacer during knee resection arthroplasty. However, its limitations need to be considered.

a. First, although fourteen studies were included in this review, no controlled trials were identified.

b. Most studies were retrospective case series; therefore, they were level IV studies.

c. The reviewed studies have a different length of followup.

d. The included studies have a low number of patients. The patients' characteristics, the autoclaving procedure the 
cement spacer features differed across the reviewed studies.

e. The antibiotic treatment performed after the prosthesis removal was not standardized among the studies; this feature could significantly influence the outcomes of a two-stage revision strategy.

f. Several papers do not detail the adopted autoclaving protocol; it is impossible to assess if a different autoclaving protocol could influence the re-infection rate. Future studies are needed to define a standardized autoclaving protocol.

\section{Conclusion}

The intraoperative autoclaving and re-use of a removed infected prosthesis, as a spacer, during a knee resection arthroplasty performed for PJI is an effective procedure in the management of knee PJI. This procedure has a reported re-infection rate ranging from 2.27 to $37 \%$ and a cumulative re-infection rate of $13.7 \%$ [14-16, 22-29, 33-35]; no significant differences between patients treated with autoclaved components and those with sterile static spacers were founded in the reviewed comparative studies.

The final ROM in patients treated using the autoclaved components as a spacer, compared with patients receiving static spacers, was significantly higher in three out of four comparative studies.

However, no prospective randomized controlled trials have focused on this subject; therefore, the data showed in this review have a moderate level of evidence.

Funding Open access funding provided by Università degli Studi di Bari Aldo Moro within the CRUI-CARE Agreement. The authors received no financial support for the research, authorship and/or publication of this article.

\section{Declarations}

Conflict of interest The authors declared no potential conflicts of interest concerning the research, authorship, and/or publication of this article.

Open Access This article is licensed under a Creative Commons Attribution 4.0 International License, which permits use, sharing, adaptation, distribution and reproduction in any medium or format, as long as you give appropriate credit to the original author(s) and the source, provide a link to the Creative Commons licence, and indicate if changes were made. The images or other third party material in this article are included in the article's Creative Commons licence, unless indicated otherwise in a credit line to the material. If material is not included in the article's Creative Commons licence and your intended use is not permitted by statutory regulation or exceeds the permitted use, you will need to obtain permission directly from the copyright holder. To view a copy of this licence, visit http://creativecommons.org/licenses/by/4.0/.

\section{References}

1. Bozic KJ, Kurtz SM, Lau E, Ong K, Chiu V, Vail TP et al (2010) The epidemiology of revision total knee arthroplasty in the United States. Clin Orthop Relat Res 468:45-51

2. Bozic KJ, Kurtz SM, Lau E, Ong K, Vail TP, Berry DJ (2009) The epidemiology of revision total hip arthroplasty in the United States. J Bone Joint Surg Am 91:128-133

3. Ting NT, Della Valle CJ (2017) Diagnosis of periprosthetic joint infection. An algorithm-based approach. J Arthroplasty 32:2047-2050

4. Shahi A, Parvizi J (2016) The role of biomarkers in the diagnosis of periprosthetic joint infection. EFORT Open Rev 1:275-278

5. Fehring TK, Odum SM, Troyer JL et al (2010) Joint replacement access in 2016: a supply side crisis. J Arthroplasty 25(8):1175-1181

6. Parvizi J (2017) Periprosthetic joint infection: the current hot topic. J Arthroplasty 32:2039

7. Andersson AE, Bergh I, Karlsson J, Nilsson K (2010) Patients' experiences of acquiring a deep surgical site infection: an interview study. Am J Infect Control 38(9):711-717

8. Kunutsor SK, Whitehouse MR, Blom AW, Beswick AD (2015) INFORM Team. Re-Infection outcomes following one- and twostage surgical revision of infected hip prosthesis: a systematic review and meta-analysis. PLoS ONE 10(9):e0139166

9. Zimmerli W, Trampuz A, Ochsner PE (2004) Prosthetic-joint infections. N Engl J Med 351(16):1645-1654

10. Leone JM, Hanssen AD (2005) Management of infection at the site of a total knee arthroplasty. J Bone Joint Surg Am 87(10):2335-2348

11. Hsu YC, Cheng HC, Ng TP, Chiu KY (2007) Antibiotic-loaded cement articulating spacer for 2-stage reimplantation in infected total knee arthroplasty: a simple and economic method. J Arthroplasty 22(7):1060-1066

12. Lange J, Troelsen A, Thomsen RW, Søballe K (2012) Chronic infections in hip arthroplasties: comparing risk of reinfection following one-stage and two-stage revision: a systematic review and meta-analysis. Clin Epidemiol 4:57-73

13. Kunutsor SK, Whitehouse MR, Lenguerrand E, Blom AW et al (2016) Re-Infection outcomes following one and two-stage surgical revision of infected knee prosthesis: a systematic review and meta-analysis. PLoS ONE 11(3):e0151537

14. Cuckler J (2005) The infected total knee: management options. J Arthroplasty 20:33-36

15. Emerson RH Jr, Muncie M, Tarbox TR, Higgins LL (2002) Comparison of a static with a mobile spacer in total knee infection. Clin Orthop Relat Res 404:132-138

16. Lee BJ, Kyung HS, Yoon SD (2015) Two-stage revision for infected total knee arthroplasty: based on autoclaving the recycled femoral component and intraoperative molding using antibiotic-impregnated cement on the tibial side. Clin Orthop Surg $7: 310-317$

17. Hwang JK, Oh CW, Lee HJ, Kyung HS (2009) An articulating versus non-articulating spacer for two-stage reimplantation patients who undergo in infected total knee arthroplasty. $\mathrm{J}$ Korean Knee Soc 21(3):150-157

18. Hofmann AA, Kane KR, Tkach TK, Plaster RL, Camargo MP (1995) Treatment of infected total knee arthroplasty using an articulating spacer. Clin Orthop Relat Res 321:45-54

19. Higgins JPT, Green S (2011) Cochrane handbook for systematic reviews of interventions version 5.1.0. The cochrane collaboration

20. Moher D, Liberati A, Tetzlaff J, Altman DG (2009) Preferred reporting items for systematic reviews and meta-analyses: the PRISMA statement. J ClinEpidemiol 62:1006-1012 
21. Liberati A, Altman DG, Tetzlaff J, Mulrow C, Gøtzsche PC, Ioannidis JPA et al (2009) The PRISMA statement for reporting systematic reviews and meta-analyses of studies that evaluate healthcare interventions: explanation and elaboration. BMJ 339:b2700

22. Hofmann AA, Goldberg T, Tanner AM, Kurtin SM (2005) Treatment of infected total knee arthroplasty using an articulating spacer: 2- to 12-year experience. Clin Orthop Relat Res 430:125-131

23. Huang HT, Su JY, Chen SK (2006) The results of articulating spacer technique for infected total knee arthroplasty. J Arthroplasty 21(8):1163-1168

24. Jamsen E, Sheng P, Halonen P et al (2006) Spacer prostheses in two-stage revision of infected knee arthroplasty. Int Orthop 30(4):257-261

25. Pietsch M, Hofmann S, Wenisch C (2006) Treatment of deep infection of total knee arthroplasty using a two-stage procedure. Oper Orthop Traumatol 18(1):66-87

26. Anderson JA, Sculco PK, Heitkemper S et al (2009) An articulating spacer to treat and mobilize patients with infected total knee arthroplasty. J Arthroplasty 24(4):631-635

27. Kalore NV, Maheshwari A, Sharma A, Cheng E, Gioe TJ (2012) Is there a preferred articulating spacer technique for infected knee arthroplasty? A preliminary study. Clin Orthop Relat Res 470(1):228-235

28. Kim YS, Bae KC, Cho CH, Lee KJ, Sohn ES, Kim BS (2013) Two-stage revision using a modified articulating spacer in infected total knee arthroplasty. Knee Surg Relat Res 25(4):180-185

29. Chen YP, Wu CC, Ho WP (2016) Autoclaved metal-on-cement spacer versus static spacer in two-stage revision in periprosthetic knee infection. Indian J Orthop 50(2):146-153

30. Lyons ST, Wright CA, Krute CN, Rivera FE (2016) Confirming sterility of an autoclaved infected femoral component for use in an articulated antibiotic knee spacer: a pilot study. J Arthroplasty 31(1):245-249

31. Centres for Disease Control and Prevention (CDC) Guideline for disinfection and sterilization in healthcare facilities tables. www. cdc.gov/hicpac/Disinfection_Sterilization/table_7.html\#8

32. Centres for Disease Control and Prevention (CDC) Guideline for disinfection and sterilization in healthcare facilities full PDF. http://www.cdc.gov/hicpac/pdf/guidelines/Disinfection_Nov_ 2008.pdf

33. Nodzo SR, Boyle KK, Spiro S, Nocon AA, Miller AO, Westrich GH (2017) Success rates, characteristics, and costs of articulating antibiotic spacers for total knee periprosthetic joint infection. Knee 24(5):1175-1181. https://doi.org/10.1016/j.knee.2017.05. 016

34. Goltz DE, Sutter EG, Bolognesi MP, Wellman SS (2018) Outcomes of Articulating Spacers With Autoclaved Femoral Components in Total Knee Arthroplasty Infection. J Arthroplasty 33:2595-2604. https://doi.org/10.1016/j.arth.2018.03.059

35. Karas V, Rutherford RW, Herschmiller TA, Plate JF, Bolognesi MP, Joyce MJ, Wellman SS (2020) Flash sterilization and component reimplantation is a viable option for articulating antibiotic spacers in periprosthetic knee infections. J Knee Surg. https://doi. org/10.1055/s-0040-1701518
36. Park HJ, Kim HJ, Kim S, Kim SM, Mun JU, Kim J, Kyung HS (2018) Safety of temporary use of recycled autoclaved femoral components in infected total knee arthroplasty: confirming sterility using a sonication method. Clin Orthop Surg 10(4):427-432. https://doi.org/10.4055/cios.2018.10.4.427

37. Demitri S, Vicenti G, Carrozzo M, Bizzoca D, De Franceschi D, Moretti B (2018) The Masquelet technique in the treatment of a non-infected open complex fracture of the distal tibia with severe bone and soft tissue loss: A case report. Injury 49(Suppl 4):S58 S62. https://doi.org/10.1016/j.injury.2018.11.039

38. Vicenti G, Bizzoca D, Nappi V, Pesce V, Solarino G, Carrozzo M, Moretti F, Dicuonzo F, Moretti B (2019) Serum biomarkers in the diagnosis of periprosthetic joint infection: consolidated evidence and recent developments. Eur Rev Med Pharmacol Sci 23(2 Suppl):43-50. https://doi.org/10.26355/eurrev_201904_17473

39. Vicenti G, Pesce V, Bizzoca D, Nappi V, Palmiotto F, Carrozzo M, Moretti B (2017) Perioperative plasmatic presepsin levels in patients undergoing total hip or knee replacement: a preliminary study. J Biol Regul Homeost Agents 31(4):1081-1086

40. Vicenti G, Bizzoca D, Carrozzo M, Solarino G, Moretti B (2018) Multi-omics analysis of synovial fluid: a promising approach in the study of osteoarthritis. J Biol Regul Homeost Agents 32(6 Suppl. 1):9-13

41. Mussa M, Manciulli T, Corbella M et al (2020) Epidemiology and microbiology of prosthetic joint infections: a nine-year, singlecenter experience in Pavia. Northern Italy Musculoskelet Surg. https://doi.org/10.1007/s12306-020-00638-y

42. Zanirato A, Formica M, Cavagnaro L et al (2020) Metaphyseal cones and sleeves in revision total knee arthroplasty: two sides of the same coin? Complications, clinical and radiological results-a systematic review of the literature. Musculoskelet Surg 104:2535. https://doi.org/10.1007/s12306-019-00598-y

43. Lombardo DJ, Siljander MP, Sobh A et al (2020) Periprosthetic fractures about total knee arthroplasty. Musculoskelet Surg 104:135-143. https://doi.org/10.1007/s12306-019-00628-9

44. Giannotti S, Sacchetti F, Citarelli C et al (2020) Single-use, patient-specific instrumentation technology in knee arthroplasty: a comparative study between standard instrumentation and PSI efficiency system. Musculoskelet Surg 104:195-200. https://doi. org/10.1007/s12306-019-00612-3

45. Frassanito L, Vergari A, Nestorini R et al (2020) Enhanced recovery after surgery (ERAS) in hip and knee replacement surgery: description of a multidisciplinary program to improve management of the patients undergoing major orthopedic surgery. Musculoskelet Surg 104:87-92. https://doi.org/10.1007/ s12306-019-00603-4

Publisher's Note Springer Nature remains neutral with regard to jurisdictional claims in published maps and institutional affiliations. 